\title{
Effect of Different Intercrops on Growth Parameters of Sole and Intercropped Transplanted Pigeonpea
}

\author{
N. Manjunatha*, A. S. Halepyati, B. M. Chittapur, B. G. Mastanreddy, \\ A. Amaregouda and K. Narayana Rao \\ Department of Agronomy, College of Agriculture, University of Agricultural Sciences, \\ Raichur-584 101, India \\ *Corresponding author
}

Keywords

Transplanted pigeonpea, Plant height, Leaf area, Leaf area index and leaf area duration

Article Info

Accepted:

07 November 2019

Available Online:

10 December 2019

\section{A B S T R A C T}

A filed experiment was conducted to study the "effect of different intercrops on growth parameters of sole and intercropped transplanted pigeonpea" at ICAR-KVK farm, Kalaburgi, Karnataka on vertisols medium black soils during kharif 2016-17 and 2017-18. The experiment was laid out using RCBD, replicated thrice with eleven treatments combinations. The treatments consisted of transplanted pigeonpea with greengram (1:4), transplanted pigeonpea with blackgram (1:4), transplanted pigeonpea with soybean (1:4), transplanted pigeonpea with sesamum (1:4), transplanted pigeonpea with sunflower (1:1), sole transplanted pigeonpea, sole greengram, sole blackgram, sole soybean and sole sunflower. Results of pooled data of two years revealed that sole transplanted pigeonpea recorded higher growth parameters viz., plant height $(195.99 \mathrm{~cm})$, number of leaves per plant $(605.10)$, leaf area $\left(93.81 \mathrm{dm}^{2}\right.$ plant $\left.^{-1}\right)$, leaf area index (0.834), leaf area duration (142.23 days) and total dry matter production (710.86 $\left.\mathrm{g} \mathrm{plant}^{-1}\right)$ at harvest over intercropped transplanted pigeonpea.

\section{Introduction}

Pigeonpea (Cajanus cajan (L.) Millsp.) is one of the protein rich pulse crop of the semi-arid tropics, grown predominantly under rainfed conditions. It improves soil conditions through addition of leaf fall and its deep and strong root system breaks the plough pans and improves the soil structure. Hence, it is often called a "biological plough". In India, pigeonpea occupies an area of about 3.47 million ha with a total production of 2.55 million tonnes, with an average productivity of $711 \mathrm{~kg} \mathrm{ha}^{-1}$ (Sujatha and Babalad, 2018). Pigeonpea is largely grown in Northern parts of Karnataka, especially in Kalburgi, 
Vijayapur, Bidar and Raichur districts. In dry farming areas of northern Karnataka, the rainfall is not only scanty but also erratic. Thus, soil moisture becomes the most limiting factor in pigeonpea production. In order to ensure timely sowing under delayed onset of monsoon, the transplanting of pigeonpea seedlings will be one of the best agronomic measures to overcome delayed sowing (Rajesh et al., 2013). In recent years transplanted method of pigeonpea cultivation is most popular practices in Zone I and Zone II of the agro-climatic region of northern Karnataka, in parts of Bidar, Kalaburagi and in some parts of Raichur district. The main reason for transplanting technology being popular among the small, marginal and medium land holding farmers in the region because of increased yield levels, advanced or timely sowing, efficient utilization of vertical and horizontal space, moisture, nutrients and light energy.

The initial slow growth rate and deep root system of pigeonpea offers good scope for intercropping with fast growing early maturing and shallow rooted crops. Several short duration crops mainly greengram, blackgram, soybean, groundnut, maize, sorghum pearlmillet and other crops could be grown as intercrop in long duration pigeonpea for increasing productivity and maintaining soil fertility. Intercropping of short duration legumes and pulses not only enhance the productivity but also the net returns over sole pigeonpea.

\section{Materials and Methods}

A field experiment was conducted kharif seasons of 2016-17 and 2017-18 at ICARKVK, Kalaburgi, and Karnataka, India. The soil reaction of the experimental field was 8.5 $(\mathrm{pH})$ with clay loam in texture, low in organic carbon $(0.45 \%)$ and available nitrogen (178 $\left.\mathrm{kg} \mathrm{ha}{ }^{-1}\right)$, medium in available phosphorus $\left(26.7 \mathrm{~kg} \mathrm{ha}^{-1}\right)$ and high in available potash $\left(506 \mathrm{~kg} \mathrm{ha}^{-1}\right)$. The experiment was laid out in randomized complete block design comprising eleven treatment combinations viz., transplanted pigeonpea with greengram (1:4 row ratio), transplanted pigeonpea with blackgram (1:4 row ratio), transplanted pigeonpea with soybean (1:4 row ratio), transplanted pigeonpea with sesamum (1:4 row ratio), transplanted pigeonpea with sunflower (1:1 row ratio), sole transplanted pigeonpea, sole greengram, sole blackgram, sole soybean and sole sunflower with three replications. The varieties used were BSMR736 of pigeonpea, BGS-9 of greengram, DU-1 of blackgram, JS-335 of soybean, DS-5 of sesamum and RSFH-1887 of sunflower. The recommended doses of fertilizers were applied for each sole crop as per the recommendations. In case of intercropping treatments, fertilizers were applied in proportionate to the sole optimum population for main and intercrops separately. The other management operations were done as per recommended package of practices for both main and intercrops. Growth parameters were recorded as per standard procedures. The height of the plant was measured from ground level to the tip of the main shoot. The plant height was measured at 75,120, 165 DAS and at harvest. The mean of five plants in each plot was worked out and expressed in $\mathrm{cm}^{\text {plant }}{ }^{-1}$.

Total number of fully opened trifoliate leaves was counted in the five plants and their average was taken as number of leaves per plant.

The leaf area was worked out by disc method on dry weight basis at 75, 120, 165 DAS and at harvest as per the procedure suggested by Vivekanandan et al., (1972).

$\mathrm{LA}=\frac{\mathrm{Wa} \times \mathrm{A}}{\mathrm{Wd}}$

Where, 
$\mathrm{LA}=$ Leaf area $\left(\mathrm{dm}^{2}\right.$ plant $\left.^{-1}\right)$

$\mathrm{Wa}=$ Oven dry weight of all leaves (inclusive of 10 disc weight)

$\mathrm{Wd}=$ Oven dry weight of 10 discs in gram

$A=$ Area of the 10 discs $\left(\mathrm{dm}^{2}\right)$

Leaf area index (LAI) was worked out by dividing the leaf area per plant by land area occupied by the plant (Sestak et al., 1971).

$\mathrm{LAI}=\frac{\mathrm{A}}{\mathrm{P}}$

Where,

$\mathrm{A}=$ Leaf area per plant $\left(\mathrm{dm}^{2}\right)$

$\mathrm{P}=$ Land area occupied by the plant $\left(\mathrm{dm}^{2}\right)$

Leaf area duration (LAD) an integral of leaf area index over the growth period was worked out using the formula given by Power et al., (1967).

$\mathrm{LAD}=\frac{\left[\mathrm{L}_{1}+\mathrm{L}_{2}\right]}{2} \times\left[\mathrm{t}_{1}-\mathrm{t}_{2}\right]$

Where,

$\mathrm{LAD}=$ Leaf area duration in days

$\mathrm{L}_{1}, \mathrm{~L}_{2}=\mathrm{LAI}$ at stage 1 and stage 2

$t_{1}$ and $t_{2}=$ Time interval in days between stage 1 and stage 2

Total dry matter was calculated by adding the dry weights of different plant parts and expressed as grams per plant.

The data were statistically analyzed as per the procedure given by Gomez and Gomez (1984) for randomized block design.

\section{Results and Discussion}

Effect of intercrops on plant height of transplanted pigeonpea

Plant height of transplanted pigeonpea differed significantly due to intercropping during both the years of experimentation and in their pooled data at all the growth stages except at 75 DAS.

At 120 DAS, among different treatments, significantly higher plant height $(134.82 \mathrm{~cm})$ was recorded with treatment $\mathrm{T}_{6}$-sole transplanted pigeonpea, but it was on par with the treatments, $\mathrm{T}_{3}$-transplanted pigeonpea + soybean (1:4 row proportion), $\mathrm{T}_{5}$-transplanted pigeonpea + sunflower (1:1 row proportion), T4- transplanted pigeonpea + sesamum (1:4 row proportion) and $\mathrm{T}_{1^{-}}$transplanted pigeonpea + greengram (1:4 row proportion) (133.81, 131.01, 126.21 and $125.27 \mathrm{~cm}$, respectively) and significantly lower plant height was noticed with treatment $T_{2}$ transplanted pigeonpea with blackgram $(111.53 \mathrm{~cm})$ in pooled data.

At 165 DAS, pooled mean indicated that significantly higher plant height $(183.82 \mathrm{~cm})$ was recorded with sole transplanted pigeonpea ( $\left.\mathrm{T}_{6}\right)$ which was found to be on par with intercropping treatments, T3-transplanted pigeonpea + soybean (1:4 row proportion) $(179.36 \mathrm{~cm})$ and $\mathrm{T}_{5^{-}}$transplanted pigeonpea + sunflower (1:1 row proportion) $(172.62 \mathrm{~cm})$ and significantly lower plant height (143.55 $\mathrm{cm})$ was recorded with transplanted pigeonpea + blackgram (1:4 row proportion).

At harvest, sole transplanted pigeonpea $\left(\mathrm{T}_{6}\right)$ recorded significantly higher plant height $(195.99 \mathrm{~cm})$, which was on par with treatments, $\mathrm{T}_{3}$-transplanted pigeonpea+ soybean (1:4 row proportion), $\mathrm{T}_{5}$-transplanted pigeonpea + sunflower $(1: 1), \mathrm{T}_{4}$-transplanted pigeonpea + sesamum (1:4 row proportion) 
and $\mathrm{T}_{1}$-transplanted pigeonpea + greengram (1:4row proportion) (190.31, 182.09, 176.11 and $174.58 \mathrm{~cm}$, respectively). Significantly lower plant height was recorded with $\mathrm{T}_{2^{-}}$ transplanted pigeonpea +blackgram (157.26 cm) (Table 1).

\section{Effect of intercrops on number of leaves of transplanted pigeonpea}

At 75 DAS, pooled data indicated that significantly higher number of leaves was recorded with $\mathrm{T}_{6}$ sole transplanted pigeonpea (50.93) as compared to intercropped treatments. Among the intercropping treatments highest number of leaves was recorded with transplanted pigeonpea + soybean (1:4) (39.23). Significantly lower number of leaves (18.83 plant $\left.^{-1}\right)$ was observed with transplanted pigeonpea + blackgram (1:4 row proportion).

At $120 \mathrm{DAS}, \mathrm{T}_{6}$ - sole transplanted pigeonpea recorded significantly higher number of leaves (542.20 plant $\left.^{-1}\right)$ over the intercropping systems. Significantly lower number of leaves plant $^{-1}$ were noticed with $\mathrm{T}_{2}$-transplanted pigeonpea + blackgram (1:4 row proportion) (205.73) in pooled data.

At 165 DAS, pooled data of two years revealed that the number of leaves plant ${ }^{-1}$ were significantly higher with $\mathrm{T}_{6}$-sole transplanted pigeonpea (1334.50) and among the intercropping treatments, $\mathrm{T}_{3}$-transplanted pigeonpea + soybean recorded higher number of higher number leaves (952.67 plant $^{-1}$ ). Significantly lower numbers of leaves were noticed with $\mathrm{T}_{2}$-transplanted pigeonpea + blackgram (1:4 row proportion) (441.17 plant $^{-1}$ ).

At harvest, significantly higher number of leaves plant $^{-1}$ was recorded with $\mathrm{T}_{6}$-sole transplanted pigeonpea (605.10) as compared to intercropped treatments. Significantly lower number of leaves plant ${ }^{-1}$ were-noticed with $\mathrm{T}_{2}$ transplanted pigeonpea + blackgram (1:4 row proportion) (212.70) from the pooled mean (Table 2).

\section{Effect of intercrops on leaf area of transplanted pigeonpea}

Pooled data at 75 DAS, revealed that the leaf area was significantly higher in the $\mathrm{T}_{6}$-sole transplanted pigeonpea $\left(7.87 \mathrm{dm}^{2}\right.$ plant $\left.^{-1}\right)$ over the intercropping treatments. Among the intercropping treatments, higher leaf area plant $^{-1}$ was noticed with $\mathrm{T}_{3^{-}}$transplanted pigeonpea + soybean (1:4 row proportion) $\left(6.14 \mathrm{dm}^{2}\right.$ plant $\left.^{-1}\right)$. The treatment $\mathrm{T}_{2^{-}}$ transplanted pigeonpea + blackgram (1:4 row proportion) recorded significantly lower leaf area plant ${ }^{-1}\left(2.89 \mathrm{dm}^{2}\right.$ plant $\left.^{-1}\right)$.

At 120 DAS, among the different treatments, significantly higher leaf area was noticed with $\mathrm{T}_{6}$-sole transplanted pigeonpea $\left(103.90 \mathrm{dm}^{2}\right.$ plant $\left.^{-1}\right)$. However, among the intercropping system treatments, $\mathrm{T}_{3^{-}}$transplanted pigeonpea + soybean (1:4 row proportion) $\left(82.45 \mathrm{dm}^{2}\right.$ plant $\left.^{-1}\right)$ recorded higher leaf area. $\mathrm{T}_{2^{-}}$ transplanted pigeonpea + blackgram (1:4 row proportion) recorded significantly lower leaf area $\left(38.84 \mathrm{dm}^{2}\right.$ plant $\left.^{-1}\right)$ in pooled data.

Pooled data at 165 DAS, revealed that the leaf area was significantly higher with sole transplanted pigeonpea (195.66 $\mathrm{dm}^{2}$ plant $\left.^{-1}\right)$. Among the intercropping treatments, transplanted pigeonpea + soybean (1:4 row proportion) noticed higher leaf area $\left(148.35 \mathrm{dm}^{2} \quad\right.$ plant $\left.^{-1}\right)$. Treatment $\mathrm{T}_{2^{-}}$ transplanted pigeonpea + blackgram (1:4 row proportion) recorded significantly lower leaf area $\left(68.26 \mathrm{dm}^{2}\right.$ plant $\left.^{-1}\right)$.

At harvest, pooled data on leaf area revealed that sole transplanted pigeonpea noticed significantly higher leaf area $\left(93.81 \mathrm{dm}^{2}\right.$ plant $\left.^{-1}\right)$. However, among the intercropping 
treatments, $\mathrm{T}_{3}$-transplanted pigeonpea + soybean (1:4 row proportion) recorded higher leaf area $\left(74.43 \mathrm{dm}^{2}\right.$ plant $\left.^{-1}\right)$. Significantly lower leaf area was noticed with $\mathrm{T}_{2}$ transplanted pigeonpea + blackgram (1:4 row proportion) (32.92 $\mathrm{dm}^{2}$ plant $^{-1}$ ) (Table 3$)$.

\section{Effect of intercrops on leaf area index of transplanted pigeonpea}

Pooled data at 75 DAS revealed that, significantly higher leaf area index (0.070) was noticed with $\mathrm{T}_{6}$-sole transplanted pigeonpea. Among the different intercropping system, transplanted pigeonpea + soybean (1:4 row proportion) recorded higher leaf area index (0.055). Significantly lower leaf area index was noticed with transplanted pigeonpea + blackgram (1:4 row proportion) (0.026).

Pooled data of two years at 120 DAS, revealed that, significantly higher leaf area index was noticed with $\mathrm{T}_{6}$-sole transplanted pigeonpea (0.924). Among the intercropping treatments, transplanted pigeonpea + soybean (1:4 row proportion) recorded higher leaf area index (0.733). Treatment $\mathrm{T}_{2}$-transplanted pigeonpea + blackgram (1:4 row proportion) recorded significantly lower leaf area index (0.345).

At 165 DAS, results of pooled data of two years revealed that, sole transplanted pigeonpea $\left(\mathrm{T}_{6}\right)$ noticed significantly higher leaf area index (1.739). Among the intercropping treatments, transplanted pigeonpea + soybean (1:4 row proportion) recorded higher leaf area index (1.319). Significantly lower leaf area (0.607) was noticed in transplanted pigeonpea + blackgram (1:4 row proportion).

At harvest, sole transplanted pigeonpea recorded significantly higher leaf area index (0.834) over the intercropping treatments. Among the different intercropping treatments, transplanted pigeonpea+ soybean (1:4) noticed higher leaf area index (0.662). Significantly lower leaf area index (0.293) was recorded with transplanted pigeonpea + blackgram (1:4 row proportion) (Table 4).

\section{Effect of intercrops on leaf area duration of transplanted pigeonpea}

Leaf area duration (75-120 DAS) revealed that, significantly higher leaf area duration was noticed with $\mathrm{T}_{6}$-sole transplanted pigeonpea (22.35 days) over intercropping treatments in pooled data. Among the intercropping treatments, transplanted pigeonpea+ soybean (1:4) noticed higher leaf area duration (17.72 days). Significantly lower leaf area duration was recorded with $T_{2-}$ transplanted pigeonpea + blackgram (1:4 row proportion) (8.35 days).

Pooled data of leaf area duration (120-165 DAS) revealed that significantly higher leaf area duration (59.91 days) was noticed with $\mathrm{T}_{6}$-sole transplanted pigeonpea over the intercropping treatments. The transplanted pigeonpea + soybean noticed higher leaf area duration (46.16 day) among the intercropping treatments. Significantly lower leaf area duration (21.42) was noticed with transplanted pigeonpea + blackgram (1:4 row proportion).

Leaf area duration at 165-harvest revealed that the $\mathrm{T}_{6^{-}}$sole transplanted pigeonpea recorded significantly higher leaf area duration (142.23days) over the intercropping treatments. Among the intercropping treatments, $\mathrm{T}_{3}$-transplanted pigeonpea+ soybean (1:4) recorded higher leaf area duration (109.42 days). Significantly lower leaf area duration (49.69 day) was recorded with transplanted pigeonpea + blackgram (1:4 row proportion) in pooled data (Table 5). 
Table.1 Plant height of transplanted pigeonpea at different growth stages as influenced by intercropping systems

\begin{tabular}{|c|c|c|c|c|c|c|c|c|c|c|c|c|}
\hline \multirow[t]{3}{*}{ Treatments } & \multicolumn{12}{|c|}{ Plant height ( cm ) } \\
\hline & \multicolumn{3}{|c|}{75 DAS } & \multicolumn{3}{|c|}{$120 \mathrm{DAS}$} & \multicolumn{3}{|c|}{165 DAS } & \multicolumn{3}{|c|}{ At harvest } \\
\hline & 2016 & 2017 & Pooled & 2016 & 2017 & Pooled & 2016 & 2017 & Pooled & 2016 & 2017 & Pooled \\
\hline $\mathbf{T}_{1}$ & 64.74 & 59.47 & 62.10 & 130.19 & 120.35 & 125.27 & 143.79 & 163.49 & 153.64 & 169.05 & 180.11 & 174.58 \\
\hline $\mathbf{T}_{2}$ & 64.37 & 60.60 & 61.98 & 115.26 & 107.81 & 111.53 & 130.66 & 156.45 & 143.55 & 150.61 & 163.91 & 157.26 \\
\hline $\mathbf{T}_{\mathbf{3}}$ & 68.06 & 62.40 & 65.23 & 137.69 & 129.93 & 133.81 & 162.27 & 196.45 & 179.36 & 178.59 & 202.03 & 190.31 \\
\hline $\mathbf{T}_{4}$ & 68.93 & 58.47 & 63.53 & 123.85 & 128.57 & 126.21 & 132.21 & 187.72 & 159.96 & 161.45 & 190.77 & 176.11 \\
\hline $\mathbf{T}_{5}$ & 66.09 & 69.00 & 67.55 & 132.48 & 129.54 & 131.01 & 164.41 & 180.83 & 172.62 & 174.55 & 189.62 & 182.09 \\
\hline $\mathrm{T}_{6}$ & 65.85 & 60.60 & 63.22 & 138.57 & 131.06 & 134.82 & 167.93 & 199.71 & 183.82 & 179.98 & 205.33 & 195.99 \\
\hline S.Em. \pm & 2.75 & 6.00 & 3.03 & 5.84 & 3.85 & 7.01 & 6.05 & 5.57 & 5.17 & 6.26 & 6.01 & 7.42 \\
\hline CD at $5 \%$ & NS & NS & NS & 18.39 & 12.12 & 22.09 & 19.07 & 17.54 & 16.29 & 19.73 & 18.93 & 23.39 \\
\hline
\end{tabular}

$\mathrm{T}_{1}$ : Transplanted pigeonpea + greengram (1:4) $\quad \mathrm{T}_{4}$ : Transplanted pigeonpea + sesamum (1:4)

$\mathrm{T}_{2}$ : Transplanted pigeonpea + blackgram (1:4) $\quad \mathrm{T}_{5}$ :Transplanted pigeonpea + sunflower $(1: 1)$

$\mathrm{T}_{3}$ : Transplanted pigeonpea + soybean $(1: 4) \quad \mathrm{T}_{6}$ : Sole transplanted pigeonpea

Table.2 Number of leaves of transplanted pigeonpea at different growth stages as influenced by intercropping systems

\begin{tabular}{|c|c|c|c|c|c|c|c|c|c|c|c|c|}
\hline \multirow[t]{3}{*}{ Treatments } & \multicolumn{12}{|c|}{ Number of leaves plant $^{-1}$} \\
\hline & \multicolumn{3}{|c|}{75 DAS } & \multicolumn{3}{|c|}{120 DAS } & \multicolumn{3}{|c|}{165 DAS } & \multicolumn{3}{|c|}{ At harvest } \\
\hline & 2016 & 2017 & Pooled & 2016 & 2017 & Pooled & 2016 & 2017 & Pooled & 2016 & 2017 & Pooled \\
\hline $\mathbf{T}_{1}$ & 38.27 & 12.47 & 25.37 & 262.00 & 297.00 & 279.50 & 591.40 & 630.13 & 610.77 & 257.53 & 276.13 & 266.83 \\
\hline $\mathbf{T}_{2}$ & 24.33 & 13.33 & 18.83 & 207.93 & 203.53 & 205.73 & 440.20 & 442.13 & 441.17 & 222.00 & 203.40 & 212.70 \\
\hline $\mathbf{T}_{3}$ & 48.80 & 29.67 & 39.23 & 341.27 & 464.67 & 402.97 & 910.33 & 995.00 & 952.67 & 486.40 & 467.00 & 476.70 \\
\hline $\mathbf{T}_{4}$ & 29.20 & 17.67 & 23.43 & 206.93 & 289.33 & 248.13 & 451.33 & 615.27 & 533.30 & 231.07 & 224.60 & 227.83 \\
\hline $\mathbf{T}_{5}$ & 40.13 & 23.80 & 31.97 & 321.13 & 338.80 & 329.97 & 879.87 & 646.80 & 763.33 & 442.60 & 360.80 & 401.70 \\
\hline$T_{6}$ & 58.67 & 43.20 & 50.93 & 362.53 & 721.87 & 542.20 & 1087.53 & 1581.47 & 1334.50 & 572.60 & 637.60 & 605.10 \\
\hline 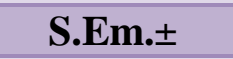 & 1.59 & 2.57 & 1.44 & 13.31 & 27.35 & 25.54 & 45.67 & 68.90 & 37.94 & 27.57 & 16.17 & 20.22 \\
\hline CD at $5 \%$ & 5.02 & 8.11 & 4.54 & 41.93 & 86.18 & 80.47 & 143.91 & 217.11 & 119.57 & 86.87 & 50.94 & 63.72 \\
\hline \multirow{2}{*}{\multicolumn{13}{|c|}{$\begin{array}{l}\mathrm{T}_{1}: \text { Transplanted pigeonpea + greengram }(1: 4) \\
\mathrm{T}_{2}: \text { Transplanted pigeonpea + blackgram }(1: 4)\end{array}$}} \\
\hline & & & & & & & & & & & & \\
\hline
\end{tabular}


Table.3 Leaf area of transplanted pigeonpea at different growth stages as influenced by intercropping systems

\begin{tabular}{|c|c|c|c|c|c|c|c|c|c|c|c|c|}
\hline \multirow[t]{3}{*}{ Treatments } & \multicolumn{12}{|c|}{ Leaf area $\left(\mathrm{dm}^{2}\right.$ plant $\left.^{-1}\right)$} \\
\hline & \multicolumn{3}{|c|}{75 DAS } & \multicolumn{3}{|c|}{120 DAS } & \multicolumn{3}{|c|}{165 DAS } & \multicolumn{3}{|c|}{ At harvest } \\
\hline & 2016 & 2017 & Pooled & 2016 & 2017 & Pooled & 2016 & 2017 & Pooled & 2016 & 2017 & Pooled \\
\hline $\mathbf{T}_{1}$ & 5.62 & 2.51 & 4.07 & 45.73 & 59.63 & 52.68 & 92.85 & 96.82 & 94.83 & 39.91 & 42.57 & 41.24 \\
\hline $\mathbf{T}_{2}$ & 3.54 & 2.24 & 2.89 & 36.96 & 40.71 & 38.84 & 72.45 & 67.41 & 68.26 & 34.47 & 31.38 & 32.92 \\
\hline $\mathbf{T}_{\mathbf{3}}$ & 7.17 & 5.11 & 6.14 & 71.86 & 93.05 & 82.45 & 142.92 & 153.78 & 148.35 & 75.33 & 73.53 & 74.43 \\
\hline $\mathbf{T}_{4}$ & 4.30 & 2.91 & 3.61 & 37.00 & 57.92 & 47.46 & 70.86 & 95.15 & 83.01 & 35.81 & 35.24 & 35.53 \\
\hline $\mathbf{T}_{5}$ & 5.90 & 3.94 & 4.92 & 63.26 & 67.78 & 65.52 & 138.14 & 100.68 & 119.41 & 68.48 & 56.73 & 62.61 \\
\hline $\mathbf{T}_{6}$ & 8.61 & 7.13 & 7.87 & 78.62 & 129.17 & 103.90 & 170.74 & 220.58 & 195.66 & 88.68 & 98.93 & 93.81 \\
\hline S.Em. \pm & 0.23 & 0.37 & 0.22 & 3.63 & 4.18 & 2.38 & 6.67 & 3.85 & 5.77 & 4.24 & 3.60 & 1.73 \\
\hline CD at $5 \%$ & 0.74 & 1.17 & 0.68 & 11.45 & 13.16 & 7.52 & 21.01 & 12.14 & 18.20 & 13.36 & 11.35 & 5.47 \\
\hline
\end{tabular}

$\mathrm{T}_{1}$ : Transplanted pigeonpea + greengram (1:4) $\mathrm{T}_{4}$ : Transplanted pigeonpea + sesamum (1:4)

$\mathrm{T}_{2}$ : Transplanted pigeonpea + blackgram (1:4) $\quad \mathrm{T}_{5}:$ Transplanted pigeonpea + sunflower $(1: 1)$

$\mathrm{T}_{3}$ : Transplanted pigeonpea + soybean $(1: 4) \quad \mathrm{T}_{6}$ : Sole transplanted pigeonpea

Table.4 Leaf area index of transplanted pigeonpea at different growth stages as influenced by intercropping systems

\begin{tabular}{|c|c|c|c|c|c|c|c|c|c|c|c|c|}
\hline \multirow[t]{3}{*}{ Treatments } & \multicolumn{12}{|c|}{ Leaf area index } \\
\hline & \multicolumn{3}{|c|}{75 DAS } & \multicolumn{3}{|c|}{120 DAS } & \multicolumn{3}{|c|}{165 DAS } & \multicolumn{3}{|c|}{ At harvest } \\
\hline & 2016 & 2017 & Pooled & 2016 & 2017 & Pooled & 2016 & 2017 & Pooled & 2016 & 2017 & Pooled \\
\hline $\mathbf{T}_{1}$ & 0.050 & 0.022 & 0.036 & 0.406 & 0.530 & 0.468 & 0.825 & 0.86 & 0.843 & 0.355 & 0.378 & 0.367 \\
\hline $\mathbf{T}_{2}$ & 0.031 & 0.020 & 0.026 & 0.329 & 0.362 & 0.345 & 0.614 & 0.60 & 0.607 & 0.306 & 0.279 & 0.293 \\
\hline $\mathbf{T}_{3}$ & 0.064 & 0.045 & 0.055 & 0.639 & 0.827 & 0.733 & 1.270 & 1.37 & 1.319 & 0.670 & 0.654 & 0.662 \\
\hline $\mathbf{T}_{4}$ & 0.038 & 0.026 & 0.032 & 0.329 & 0.515 & 0.422 & 0.630 & 0.85 & 0.738 & 0.318 & 0.313 & 0.316 \\
\hline $\mathbf{T}_{5}$ & 0.052 & 0.035 & 0.044 & 0.562 & 0.602 & 0.582 & 1.228 & 0.89 & 1.061 & 0.609 & 0.504 & 0.557 \\
\hline$T_{6}$ & 0.077 & 0.063 & 0.070 & 0.699 & 1.148 & 0.924 & 1.518 & 1.96 & 1.739 & 0.788 & 0.879 & 0.834 \\
\hline S.Em. \pm & 0.002 & 0.003 & 0.002 & 0.033 & 0.037 & 0.021 & 0.035 & 0.11 & 0.037 & 0.038 & 0.015 & 0.015 \\
\hline CD at $5 \%$ & 0.007 & 0.010 & 0.006 & 0.103 & 0.117 & 0.067 & 0.111 & 0.34 & 0.117 & 0.119 & 0.048 & 0.049 \\
\hline
\end{tabular}

$\mathrm{T}_{1}$ : Transplanted pigeonpea + greengram (1:4) $\quad \mathrm{T}_{4}$ : Transplanted pigeonpea + sesamum (1:4)

$\mathrm{T}_{2}$ : Transplanted pigeonpea + blackgram (1:4) $\quad \mathrm{T}_{5}$ : Transplanted pigeonpea + sunflower $(1: 1)$

$\mathrm{T}_{3}$ : Transplanted pigeonpea + soybean (1:4) $\quad \mathrm{T}_{6}$ : Sole transplanted pigeonpea 
Table.5 Leaf area duration of transplanted pigeonpea at different growth stages as influenced by intercropping systems

\begin{tabular}{|c|c|c|c|c|c|c|c|c|c|}
\hline \multirow[t]{3}{*}{ Treatments } & \multicolumn{9}{|c|}{ Leaf area duration(days) } \\
\hline & \multicolumn{3}{|c|}{$\begin{array}{c}\text { Leaf area duration } \\
(75-120 \text { DAS })\end{array}$} & \multicolumn{3}{|c|}{$\begin{array}{l}\text { Leaf area duration } \\
(120-165 \text { DAS })\end{array}$} & \multicolumn{3}{|c|}{$\begin{array}{c}\text { Leaf area duration } \\
\text { (165- harvest) }\end{array}$} \\
\hline & 2016 & 2017 & Pooled & 2016 & 2017 & Pooled & 2016 & 2017 & Pooled \\
\hline$T_{1}:$ Transplanted pigeonpea + greengram (1:4) & 10.27 & 12.43 & 11.35 & 27.72 & 31.29 & 29.50 & 64.90 & 68.76 & 66.83 \\
\hline$T_{2}:$ Transplanted pigeonpea + blackgram (1:4) & 8.10 & 8.59 & 8.35 & 21.21 & 21.62 & 21.42 & 50.64 & 48.73 & 49.69 \\
\hline$T_{3}:$ Transplanted pigeonpea + soybean $(1: 4)$ & 15.80 & 19.63 & 17.72 & 42.96 & 49.37 & 46.16 & 106.70 & 112.14 & 109.42 \\
\hline$T_{4}:$ Transplanted pigeonpea + sesamum $(1: 4)$ & 8.26 & 12.17 & 10.21 & 21.57 & 30.61 & 26.09 & 52.15 & 64.33 & 58.24 \\
\hline$T_{5}:$ Transplanted pigeonpea + sunflower $(1: 1)$ & 13.83 & 14.34 & 14.09 & 40.28 & 33.69 & 36.99 & 101.02 & 77.66 & 89.34 \\
\hline$T_{6}:$ Sole transplanted pigeonpea & 17.45 & 27.26 & 22.35 & 49.87 & 69.95 & 59.91 & 126.83 & 157.63 & 142.23 \\
\hline S.Em. \pm & 0.40 & 0.81 & 0.47 & 1.11 & 1.15 & 1.26 & 3.80 & 3.04 & 3.22 \\
\hline CD at $5 \%$ & 1.27 & 2.57 & 1.49 & 3.49 & 3.63 & 3.96 & 11.98 & 9.59 & 10.14 \\
\hline
\end{tabular}

Table.6 Total dry matter production of transplanted pigeonpea at different growth stages as influenced by intercropping systems

\begin{tabular}{|c|c|c|c|c|c|c|c|c|c|c|c|c|}
\hline \multirow[t]{3}{*}{ Treatments } & \multicolumn{12}{|c|}{ Total dry matter production (g plant ${ }^{-1}$ ) } \\
\hline & \multicolumn{3}{|c|}{75 DAS } & \multicolumn{3}{|c|}{120 DAS } & \multicolumn{3}{|c|}{165 DAS } & \multicolumn{3}{|c|}{ At harvest } \\
\hline & 2016 & 2017 & Pooled & 2016 & 2017 & Pooled & 2016 & 2017 & Pooled & 2016 & 2017 & Pooled \\
\hline $\mathbf{T}_{1}$ & 22.43 & 21.97 & 22.20 & 111.53 & 115.03 & 113.28 & 198.90 & 209.57 & 204.23 & 462.91 & 547.17 & 505.04 \\
\hline $\mathbf{T}_{2}$ & 18.90 & 18.70 & 18.80 & 87.80 & 72.43 & 80.11 & 131.87 & 152.70 & 142.28 & 390.87 & 321.93 & 356.40 \\
\hline $\mathbf{T}_{\mathbf{3}}$ & 33.52 & 49.24 & 41.38 & 167.73 & 171.57 & 169.65 & 304.47 & 334.53 & 319.50 & 614.60 & 679.73 & 647.17 \\
\hline $\mathbf{T}_{4}$ & 18.93 & 24.30 & 21.62 & 83.47 & 103.93 & 93.53 & 127.80 & 196.77 & 162.28 & 362.40 & 445.78 & 404.09 \\
\hline $\mathbf{T}_{5}$ & 29.80 & 38.80 & 34.30 & 157.10 & 161.45 & 159.27 & 278.37 & 311.43 & 294.90 & 519.73 & 650.49 & 585.11 \\
\hline $\mathbf{T}_{6}$ & 43.10 & 56.30 & 49.70 & 180.90 & 214.58 & 197.74 & 323.93 & 401.43 & 362.68 & 642.40 & 779.32 & 710.86 \\
\hline S.Em. \pm & 1.51 & 1.66 & 1.05 & 11.21 & 7.33 & 4.06 & 13.59 & 8.42 & 8.58 & 46.49 & 30.55 & 24.97 \\
\hline CD at $5 \%$ & 4.75 & 5.22 & 3.32 & 35.32 & 23.10 & 12.81 & 42.82 & 26.54 & 27.04 & 146.50 & 96.25 & 78.69 \\
\hline
\end{tabular}

$\mathrm{T}_{1}$ : Transplanted pigeonpea + greengram (1:4) $\quad \mathrm{T}_{4}$ : Transplanted pigeonpea + sesamum (1:4)

$\mathrm{T}_{2}$ : Transplanted pigeonpea + blackgram (1:4) $\quad \mathrm{T}_{5}:$ Transplanted pigeonpea + sunflower $(1: 1)$

$\mathrm{T}_{3}$ : Transplanted pigeonpea + soybean $(1: 4) \quad \mathrm{T}_{6}$ : Sole transplanted pigeonpea 
Effect of intercrops on the total dry matter production of transplanted pigeonpea

Pooled data at 75 DAS, revealed that, significantly higher total dry matter production was recorded with sole transplanted pigeonpea $\left(49.70 \mathrm{~g} \mathrm{plant}^{-1}\right)$ over other intercropping treatments. Among the intercropping treatments, $\mathrm{T}_{3}$-transplanted pigeonpea + soybean (1:4 row proportion) noticed higher total dry matter production (41.38 g plant $\left.^{-1}\right) . \mathrm{T}_{2}$ - transplanted pigeonpea + blackgram (1:4 row proportion) recorded significantly lower total dry matter production $\left(18.80 \mathrm{~g} \mathrm{plant}^{-1}\right)$.

At 120 DAS, pooled data on intercropping of transplanted pigeonpea results revealed that, significantly higher total dry matter production (197.74 $\mathrm{g}$ plant $\left.^{-1}\right)$ was recorded with $\mathrm{T}_{6}$-sole transplanted pigeonpea when compared to other intercropping treatments. Among the intercropping treatments $\mathrm{T}_{3^{-}}$ transplanted pigeonpea + soybean (1:4 row proportion) noticed higher total dry matter production (169.65 $\mathrm{g} \mathrm{plant}^{-1}$ ) compared to other intercropping treatments under study. Significantly lower total dry matter production was recorded with transplanted pigeonpea + blackgram (1:4 row proportion) (80.11 $\mathrm{g} \mathrm{plant}^{-}$ $\left.{ }^{1}\right)$. Similar trend was observed during 165 DAS also.

At harvest, sole transplanted pigeonpea $\left(\mathrm{T}_{6}\right)$ recorded significantly higher total dry matter production (710.86 $\mathrm{g} \mathrm{plant}^{-1}$ ) which was found on par with transplanted pigeonpea + soybean (1:4 row proportion) (647.17 $\left.\mathrm{g} \mathrm{plant}^{-1}\right)$. Significantly lower total dry matter production (356.40) was noticed with transplanted pigeonpea + blackgram (1:4 row proportion) in pooled data (Table 6). The above results corroborated well with Rathod et al., (2004), Shanmugam (2008), Srichandan and Mangaraj (2015), Nagar et al., (2015) and Pal et al., (2016).
Based on the results, it can be concluded that sole transplanted pigeonpea recorded higher growth parameters viz., plant height, number of leaves per plant, leaf area, leaf area index, leaf area duration and total dry matter production over intercropped transplanted pigeonpea. Among the intercropping systems, transplanted pigeonpea + soybean (1:4 row proportion) fallowed by transplanted pigeonpea + soybean (1:4 row proportion) higher growth parameter compare to other intercropping treatments.

\section{References}

Nagar, R. K., Goud, V. V., Kumar, R. and Kumar, R., 2015, Effect of incorporation of FYM, pigeonpea stalk, phosphor-compost on growth, yield and nutrient uptake in pigeonpea based intercropping system. Bioscan, 10: 339-343.

Pal, A. K., Singh, R. S., Shukla, U. N. and Singh, S., 2016, Growth and production potential of pigeonpea (Cajanus cajan L.) as influenced by intercropping and integrated nutrient management. J. App. Natu. Sci., 8: 179 -183 .

Power, J. F., Willis, W. O. and Reichman, G. A., 1967, Effect of soil temperature, $P$ and plant age on growth analysis of barley. Agron. J., 18:459-463.

Rajesh, N., Paulapandi, V. K. and Duraisingh, R., 2013, Effect of transplanted pigeonpea and foliar nutrition improves the physiological characters. Paper presented in: Nationa. Sem., Ergonomics for Enhanced Productivity, TNAU, Madurai, p. 12.

Rathod, P. S., Halikatti, S. I., Hiremath S. M., and Kajjidoni, S. T., 2004, Influence of different intercrops and row proportions on yield and yield parameters of pigeonpea in vertisols of 
Dharwad. Karnataka J.Agric.Sci., 17: 652-657.

Sestak, Z., Castsky, J. and Jarvis, P. G., 1971, Plant photosynthetic production. Mannual of methods (Ed.).W.JUNK, N. V., publication. The Hughes, pp. 343-381.

Shanmugam, P. M., 2008, Production potential and economics of pigeonpea (Cajanus cajan L.) based intercropping system with different level and forms of P. J. Fmg. Sys. Res. Dev., 14(1): $118-112$.

Srichandan, S. and Mangaraj, A. K., 2015, Growth, yield and yield attributes of pigeonpea in rainfed uplands of western central table land zone of Odisha. Int. J. Res. Agric. Fore., 2: 1013.

Sujatha, H. T. and Babalad, H. B., 2018, System productivity and economics of transplanted and direct sown pigeonpea at different cropping geometry and intercropping systems. Int. J. Pure App. Biosci., 6: 694-700.

Vivekanandan, A. S., Gounasena, H. P. M. and Shivananygan, T., 1972, Statistical evaluation of the occurring of three techniques used in the estimation of leaf area of crop plant. Indian J. Agric. Sci., 42: 857-860.

\section{How to cite this article:}

Manjunatha, N., A. S. Halepyati, B. M. Chittapur, B. G. Mastanreddy, A. Amaregouda and Narayana Rao, K. 2019. Effect of Different Intercrops on Growth Parameters of Sole and Intercropped Transplanted Pigeonpea. Int.J.Curr.Microbiol.App.Sci. 8(12): 470-479.

doi: https://doi.org/10.20546/ijcmas.2019.812.062 